\title{
Assessment of lipophilicity of newly synthesized celecoxib analogues using reversed-phase HPLC
}

\author{
Heba Elmansi ${ }^{1 *} \mathbb{D}$, Jenny Jeehan Nasr ${ }^{1 \dagger}$, Azza H. Rageh ${ }^{2 \dagger}$, Mohamed I. El-Awady ${ }^{1}$, Ghada S. Hassan ${ }^{3}$, \\ Hatem A. Abdel-Aziz ${ }^{4}$ and Fathalla Belal ${ }^{1}$
}

\begin{abstract}
Background: Lipophilicity is a physicochemical property of an essential importance in medicinal chemistry; therefore, fast and reliable measurement of lipophilicity will affect greatly the drug discovery process.

Results: A series of $\mathrm{N}$-benzenesulfonamide- $1 \mathrm{H}$-pyrazoles, oximes and hydrazones as celecoxib analogues was investigated with regard to their retention behavior using reversed-phase high performance liquid chromatography (RPHPLC). The mobile phases employed for this study consist of a mixture of water and methanol in different proportions. In addition, the stationary phase utilized for this separation was $C_{18}$ silanized silica gel and using $200 \mathrm{~nm}$ as a detection wavelength. The retention behavior of the investigated compounds was determined based on practical determination of log $k$ at different concentrations of methanol (as an organic modifier) in the mobile phase ranging from 60 to $80 \%$. It was observed that the retention of these compounds (expressed as log $k$ ) decreased in a linear manner with increasing the concentration of methanol. High correlation coefficients (more than 0.90 in most cases) were obtained for the relationship between the volume fraction of the organic solvent and the retention values represented as log $k_{w}$. A comparative evaluation was carried out between chromatographically-obtained lipophilicity parameters (represented as lipophilicity chromatographic index $\log k_{w}$ or isocratic chromatographic hydrophobicity index, $\varphi_{0}$ ) and the computationally calculated log P values (miLogP, ALOGP, ACD/Labs and ALOGPs).
\end{abstract}

Conclusion: It was found that a good correlation exists between the experimental and computed log $\mathrm{P}$ values. In the future, these results can give a deep insight about the anti-inflammatory and analgesic activity of the newly synthesized compounds.

Keywords: Lipophilicity estimation, Celecoxib analogues, HPLC, Lipophilicity chromatographic index, log $k_{w}$

\section{Introduction}

Lipophilicity is a paramount descriptor which represents an essential part in design of new medications with required biological action [1]. It is also used in quantitative structure activity relationship (QSAR) investigations. The IUPAC (International Union of Pure and Applied Chemistry) defined it as "a physicochemical property which describes a partitioning equilibrium of solute

\footnotetext{
*Correspondence: dr_heba85@hotmail.com

†Jenny Jeehan Nasr, Azza H. Rageh contributed equally to this work

${ }^{1}$ Department of Pharmaceutical Analytical Chemistry, Faculty

of Pharmacy, Mansoura University, Mansoura 35516, Egypt

Full list of author information is available at the end of the article
}

molecules between water and an immiscible organic solvent" [2]. This property is one of the leading considerations as it has an important effect on pharmacodynamic and pharmacokinetic properties of drugs [3]. It suggests how the ADME (Absorption, Distribution, Metabolism, and Elimination) features of medications will proceed, in addition to its influence on their toxicological profile [4]. For its vital role on drug discovery and design process, the estimation of lipophilicity and how to regulate it has turned out to be routine procedure in drug development field [5]. Lipophilicity is also important in demonstrating the destiny of a compound in the environment, where it influences the bioavailability and bio-concentration in 
the food chain via sorption from water, and soil or dregs, which makes it an essential issue in danger evaluation and controlling of risky resources [6].

Lipophilicity is a fundamental molecular property which is defined as the logarithm of the octanolwater partition coefficient $\left(\log P_{\text {OW }}\right)$, which is, in turn, expressed as the non-ionized drug spread ratio between the two phases of octanol and water [7]. $\log P$ measurements by different experimental protocols are illustrated in detail by the Organization for Economic Cooperation and Development (OECD) guidelines as test No. 107, which is Shake Flask method [8], and Test No. 123, which is the Slow Stirring method [9]. These traditional procedures are time-consuming, limited to extremely pure compound and need specific reagent to be performed. Hence, they have recently been replaced by more adaptable simpler methodologies which are chromatographic techniques (the OECD Test No. 117 [10]) that provide coherent results in the same $\log P$ range.

In the last few years, a great interest has been given for RP-HPLC as a tool for lipophilicity estimation and for characterization of pharmacological activity of molecules [11-13]. This is attributed to the close relationship between the retention performance of molecules in reversed phase chromatographic system and its lipophilicity [14]. The major advantages in using HPLC as a superior strategy are: smaller sample amount, high sensitivity to impurities, reproducibility and accuracy in addition to short determination time. The majority of the chromatographic systems used for measuring the lipophilicity rely on hydrophobic reversed-phase silica-based stationary phase $\left(\mathrm{C}_{8}\right.$ or $\left.\mathrm{C}_{18}\right)$ [3].

In parallel to HPLC methods, computational methodologies have also been employed. Their wide use is based on considering the industrial requests as being simple, low cost, fast, and consistent approach to deliver information for fast screening of compounds under focus [5].

Celecoxib,4-[5-(4-methylphenyl)-3-(trifluoromethyl) pyrazol-1yl]benzenesulfonamide [15] is a selective inhibitor of cyclooxygenase-2 (COX-2) enzyme that is prescribed as a non-steroidal anti-inflammatory, analgesic, and antipyretic drug [16]. Being a selective cyclooxygenase-2 inhibitor, celecoxib seems to show fewer side effects than non-selective non-steroidal anti-inflammatory agents [17]. Unlike celecoxib, these non-selective agents act as inhibitors of both COX-1 and COX-2 enzymes which increases the possibility of inducing gastric ulcers, gastric bleeding and suppressed renal functions [18]. On the other hand, celecoxib has been alerted by FDA for its adverse side effects on cardiovascular system, and that represents the main motivation for discovering novel compounds with COX-2 inhibitory activity [19].
The principal aim of the present investigation is to develop an appropriate and proficient strategy for estimating the lipophilicity of a series of recently discovered $N$-benzenesulfonamide- $1 H$-pyrazoles, oximes and hydrazones as celecoxib analogues [19] by RPHPLC method and comparing these results with those obtained from computational programs. All the compounds in this series are weakly basic in nature $\left(\mathrm{pK}_{\mathrm{a}}\right.$ of celecoxib is 11.1) [15]. The investigated compounds are named 11a, 11b, 11c, 11d, 16a, 16b, 17a, and 17b in addition to celecoxib. The chemical structures of compounds under investigation are depicted in Fig. 1. Additional file 1 included H-NMR for the newly synthesized compounds.

\section{Experimental}

Reagents and chemicals

Celecoxib of purity $99.44 \%$ was kindly provided by Amoun Pharmaceutical Co. (Cairo, Egypt). The synthesis and characterization of the studied compounds (celecoxib analogues) were previously reported [16]. HPLC grade methanol was purchased from SigmaAldrich (Seelze, Germany). Double-distilled water was utilized throughout the study and was filtered through a Millipore membrane filter of bore size $0.45 \mu \mathrm{m}$ (Additional file 1).

\section{Instrumentation and chromatographic conditions}

For chromatographic analysis, stock solutions $(200 \mu \mathrm{g} /$ $\mathrm{mL}$ ) of each of the investigated samples were prepared in methanol. HPLC measurements were performed utilizing Shimadzu Prominence HPLC system (Shimadzu Corporation, Japan) consisting of LC-20 AD pump, DGU-20 A5 degasser, CBM-20A interface, and SPD-20A UV-Vis detector with a $20 \mu \mathrm{L}$ injection loop. The column utilized for studying the chromatographic behavior of the analytes was the most frequently used for lipophilicity investigations: Waters symmetry ${ }^{\circledR} C_{18}$ column [250 mm $\times 4.6 \mathrm{~mm}$ (i.d.), $5-\mu \mathrm{m}$ particle size]. The flow rate was $1.2 \mathrm{~mL} / \mathrm{min}$ at room temperature. The retention behaviour of the analytes was investigated as a function of mobile phase composition ranging from $60-80 \%$ methanol and $40-20 \%$ water. The HPLC analyses were carried out at room temperature under isocratic conditions. Membrane filters $(0.45 \mu \mathrm{m})$ from Sartorius (Goettingen, Germany) were used for filtration of samples. The absorbance of the analytes during a chromatographic run was performed at $200 \mathrm{~nm}$. Each experiment was run in duplicate. Chromatographic data were collected using LabSolutions CS 
<smiles>Cc1ccc(-c2cc(C(F)(F)F)nn2-c2ccc(S(N)(=O)=O)cc2)cc1</smiles>

Celecoxib<smiles>[X]c1ccc(C(=O)CS(=O)(=O)Br)cc1</smiles>

$11 \mathrm{a}-\mathrm{d}$<smiles>[X]c1ccc(/C(CS(=O)(=O)c2ccccc2)=N/O)cc1</smiles>

$16 \mathrm{a}, \mathrm{b}$<smiles>[X]c1ccc(/C(CS(=O)(=O)c2ccccc2)=N/Nc2ccccc2)cc1</smiles>

$17 \mathrm{a}, \mathrm{b}$

\begin{tabular}{|c|l|l|}
\hline $\mathbf{1 1}$ & $\mathbf{X}$ & $\mathrm{Ar}$ \\
\hline $\mathbf{a}$ & $\mathrm{H}$ & $\mathrm{Ph}$ \\
\hline $\mathbf{b}$ & $\mathrm{Me}$ & $\mathrm{Ph}$ \\
\hline $\mathbf{c}$ & $\mathrm{Br}$ & $\mathrm{Ph}$ \\
\hline $\mathbf{d}$ & $\mathrm{Br}$ & $4-\mathrm{MeC}_{6} \mathrm{H}_{4^{-}}$ \\
\hline
\end{tabular}

\begin{tabular}{|c|l|}
\hline $\mathbf{1 6 , 1 7}$ & $\mathbf{X}$ \\
\hline $\mathbf{a}$ & $\mathrm{H}$ \\
\hline $\mathbf{b}$ & $\mathrm{Me}$ \\
\hline
\end{tabular}

Fig. 1 Chemical structures of the studied compounds

software. $\mathrm{H}^{1} \mathrm{NMR}$ spectra were recorded using Bruker NMR spectrometer (Bruker GmbH, Germany),

\section{Computational programs}

Computational methods were performed using CS ChemDraw Ultra software (Cambridge Soft Corporation, Cambridge, MA, USA) running under Windows 7 operating system.

Computational lipophilicity (clog P) was calculated by the Advanced Chemistry Development ACD/Labs online service (https://ilab.acdlabs.com/iLab2/), Molinspiration online service (miLOGP, performed online at http://www.molinspiration.com/) and ALOGPS 2.1 online service (ALOGPS, performed online at http:// www.vcclab.org/lab/alogps/).

\section{Results and discussion}

Theoretical aspects

It was shown from previous reports that the chromatographic systems using methanol: water as mobile phases were better for $\log k$ modeling than those containing acetonitrile as an organic modifier [4]. Moreover, it was found that the most preferred packing material for reversed-phase columns in lipophilicity assessment is $C_{18}$ 
silanized silica gel. Chromatographic techniques including HPLC are based on the determination of the retention parameters [4]. The indices of lipophilicity measured by HPLC are obtained from the logarithm of the retention factor $(\log k)$, which is calculated by the following equation:

$$
\log k=\log \frac{\mathrm{t}_{\mathrm{r}}-\mathrm{t}_{0}}{\mathrm{t}_{0}}
$$

where; $t_{R}$ is the retention time of the solute and $t_{0}$ is the retention time of unretained species [20].

$\log k$ is proved experimentally to be in a linear relationship to the volume fraction of the organic solvent in the mobile phase $(\varphi)$ according to the following linear regression equation [21]:

$$
\log k=\log \mathrm{k}_{\mathrm{w}}-\mathrm{S} \varphi
$$

Table 1 Logarithm of the retention factor $(\log k)$ on $C_{18}$ column using methanol-water system $(60-80 \%$ are

\begin{tabular}{|c|c|c|c|c|c|}
\hline \multirow[t]{2}{*}{ Compound } & \multicolumn{5}{|l|}{$\log k$} \\
\hline & $60 \%$ & $65 \%$ & $70 \%$ & $75 \%$ & $80 \%$ \\
\hline $11 a$ & 0.088 & 0.041 & -0.206 & -0.412 & -0.695 \\
\hline $11 b$ & 0.317 & 0.493 & -0.016 & -0.235 & -0.607 \\
\hline $11 \mathrm{c}$ & 0.635 & 0.501 & 0.123 & -0.036 & -0.145 \\
\hline $11 d$ & a & 0.901 & 0.364 & 0.123 & -0.041 \\
\hline $16 a$ & 0.130 & 0.053 & -0.220 & -0.424 & -0.385 \\
\hline $16 b$ & 0.481 & 0.280 & 0.150 & -0.233 & -0.198 \\
\hline $17 a$ & 0.950 & 0.824 & 0.740 & -0.12 & -0.051 \\
\hline $17 b$ & 0.847 & 0.743 & 0.611 & -0.221 & -0.410 \\
\hline Celecoxib & a & 0.950 & 0.83 & 0.530 & 0.235 \\
\hline
\end{tabular}
percentages of methanol in the mobile phase)

${ }^{a}$ Compound retained on the stationary phase where; $\log \mathrm{k}_{\mathrm{w}}$ is the intercept of the linear regression curve which reflects the lipophilicity chromatographic index and denotes the factor of retention for pure water as eluent, and the slope $(\mathrm{S})$ is extensively related to the strength of solvent or with specific hydrophobic surface area of solutes, and $\varphi$ is the portion of organic modifier volume.

Estimating $\log \mathrm{k}_{\mathrm{w}}$ directly is often very difficult, and is nearly non-feasible, because it can produce elongated retention time and also extreme peak broadening. Hence, estimating $k$ with various proportions of water-organic solvent combination as mobile phases is favored, then extrapolating the correlation between $\log k$ and percentage of organic modifier specifies the value of $\log k$ upon utilizing pure water as mobile phase [22]. $\log k$ was estimated for each of the new celecoxib analogues at different proportions of methanol. Consequently, to obtain $\log \mathrm{k}_{\mathrm{w}}$, an extrapolation to $100 \%$ of pure water was carried out. Then, linear regression analysis was performed [5]. Linear extrapolation is favored using definite ratios of organic modifier consistent with the lipophilicity level of the solutes [23].

The retention data were expressed as logarithm of the retention factor $(\log k)$ as shown in Table 1. It is clear that the lower the volume fraction of the organic modifier, the lower the elution power of the mobile phase and the higher the value of $\log k$. The highest $\log k$ values were obtained when $60 \% \mathrm{v} / \mathrm{v}$ methanol was utilized.

The chromatographic data obtained for the nine investigated compounds together with the results of linear regression analysis are listed in Table 2 and represented in Fig. 2. For all examined drugs, high values of correlation coefficients were achieved $(r>0.9098)$, with small values of standard error of estimate, which proves the high significance of Eq. (2) for determination of lipophilicity. $\mathrm{S}$ is

Table 2 Linear regression parameters between the logarithm of the retention factor (log k) and methanol volume

\begin{tabular}{|c|c|c|c|c|c|c|c|c|c|c|}
\hline \multirow[t]{2}{*}{ Compound } & \multicolumn{10}{|c|}{$\log k=\log k_{w}-S \varphi$} \\
\hline & $\log k_{w}$ & $\mathrm{SD}_{\mathrm{a}}$ & $S$ & $S D_{b}$ & $r$ & $\mathrm{R}^{2}$ & $S_{y x}$ & $F$ & $\varphi_{0}$ & Range \\
\hline $11 a$ & 2.593 & \pm 0.331 & -0.040 & \pm 0.005 & 0.9804 & 0.9610 & 0.074 & 74.10 & 64.83 & $60-80$ \\
\hline $11 \mathrm{~b}$ & 3.597 & \pm 0.826 & -0.052 & \pm 0.012 & 0.9301 & 0.8650 & 0.186 & 19.25 & 69.81 & $60-80$ \\
\hline $11 \mathrm{c}$ & 3.151 & \pm 0.359 & -0.042 & \pm 0.005 & 0.9785 & 0.9570 & 0.081 & 67.59 & 75.14 & $60-80$ \\
\hline $11 d$ & 4.784 & \pm 0.887 & -0.061 & \pm 0.012 & 0.9627 & 0.9270 & 0.136 & 25.30 & 77.99 & $65-80$ \\
\hline $16 a$ & 1.941 & \pm 0.413 & -0.030 & \pm 0.006 & 0.9475 & 0.8980 & 0.092 & 26.34 & 64.39 & $60-80$ \\
\hline $16 b$ & 2.715 & \pm 0.442 & -0.037 & \pm 0.006 & 0.9602 & 0.9220 & 0.099 & 35.47 & 72.57 & $60-80$ \\
\hline $17 a$ & 4.590 & \pm 1.091 & -0.059 & \pm 0.015 & 0.9098 & 0.8280 & 0.245 & 14.41 & 77.97 & $60-80$ \\
\hline $17 b$ & 5.182 & \pm 1.017 & -0.069 & \pm 0.014 & 0.9409 & 0.8850 & 0.229 & 23.14 & 74.52 & $60-80$ \\
\hline Celecoxib & 4.180 & \pm 0.445 & -0.048 & \pm 0.006 & 0.9847 & 0.9696 & 0.068 & 63.81 & 85.51 & $65-80$ \\
\hline
\end{tabular}
fraction $(\varphi)$

$\mathrm{SD}_{\mathrm{a}}$, standard deviation of intercept; $\mathrm{SD}_{\mathrm{b}}$, standard deviation of slope, r, correlation coefficient, $\mathrm{R}^{2}$, determination coefficient, $\mathrm{S}_{\mathrm{yx}}$, $\mathrm{Standard}$ error of estimate (SEE); $\mathrm{F}$ value of test $F$ Snedecora at $P$ value $<0.05 ; \varphi_{0}$, the hydrophobicity index 


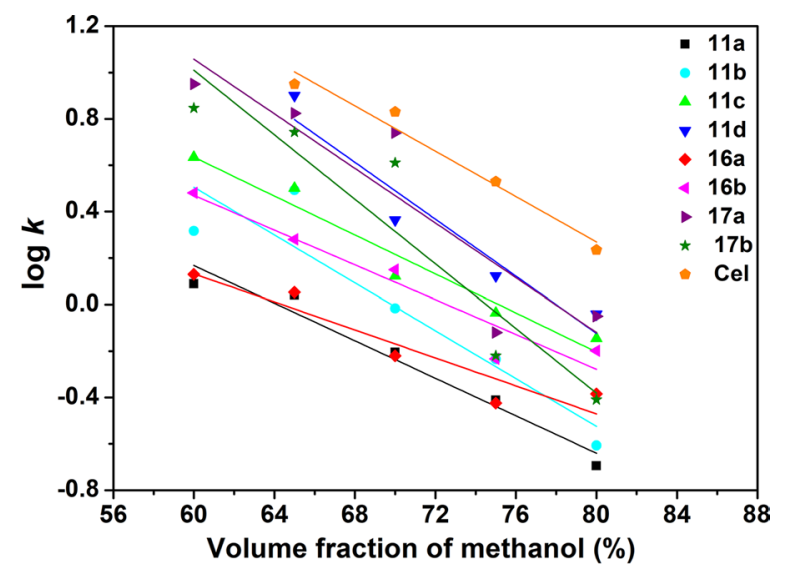

Fig. 2 Linear fitting parameters of the relationship between log $k$ and volume faction of methanol for nine of the studied compounds

related to the specific hydrophobic surface area of solutes and it relies on the solute and the chromatographic system. A good correlation exists between $\log \mathrm{k}_{\mathrm{w}}$ and slope with $r \approx-0.9716$ which suggests a similar chromatographic retention mechanism for this homologous series of studied compounds. This relationship can be represented by the following equation:

$$
\log \mathrm{k}_{\mathrm{w}}=-85.342( \pm 7.852) \mathrm{S}-0.536( \pm 0.395)
$$

$\left(r=0.9716, \quad \mathrm{R}^{2}=0.9440, \quad \mathrm{~F}=118.12, \quad \mathrm{p} \quad\right.$ value $<0.001$, $\mathrm{S}_{\mathrm{yx}}=0.2822, \mathrm{n}=9$ ), $\mathrm{R}^{2}$ is the determination coefficient, $\mathrm{F}$ is the value of test $\mathrm{F}$ - Snedecora, $\mathrm{S}_{\mathrm{yx}}$ is standard error of estimate.

Another substitute to the index of lipophilicity is the chromatographic index of hydrophobicity $\varphi_{0}$ which was first given by Valkò et al. [24]. It is calculated simply by the equation: $\varphi_{0}=-\log \mathrm{k}_{\mathrm{w}} / \mathrm{S}$. $\varphi_{0}$ denotes the volume fraction of the organic modifier in the mobile phase at which equal partitioning of the solute between the mobile and stationary phases is obtained, that is, $\mathrm{k}=1, \log k=0$ [22]. The value of $\varphi_{0}$ is specific for each compound; it relies solely on the $\mathrm{pH}$, organic modifier and temperature. It does not rely on the column type and length, flow rate and the composition of the mobile phase with which the retention determination are made. Moreover, $\varphi_{0}$ can be accurately estimated as it possesses a solid physical meaning, that is, the concentration of the organic modifier in the mobile phase where the retention time is accurately double the dead time. This is considered a distinguishable advantage over extrapolating of $\log k$ values to pure water mobile phase [24]. $\varphi_{0}$ values calculated for the studied compounds are listed in Table 2.

\section{Lipophilicity study}

Eight compounds of the N-benzenesulfonamide$1 \mathrm{H}$-pyrazoles, oximes and hydrazones series were investigated in this study as celecoxib analogues. They possess similar structural features with different substituents that significantly impact their physicochemical, pharmacokinetic, and pharmacodynamic properties. RP-HPLC was utilized to estimate their lipophilicity (conveyed as $\log \mathrm{k}_{\mathrm{w}}$ or $\left.\varphi_{0}\right)$. In this study, $\mathrm{C}_{18}$ column was utilized as the stationary phase which is consisting of silica gel amended by a lipophilic $\mathrm{C}_{18}$ hydrocarbon chain while the mobile phase was more polar than the stationary phase. It is well-known that polar molecules have lower retention times and hence lower retention factors than non-polar molecules which are strongly attracted to the stationary phase hydrocarbon groups as explained by the Van der Waals forces [25].

For the first group of the studied compounds (aryl sulfones), in relation to the retention data, it can be observed that $11 \mathrm{a}$ is more polar than $11 \mathrm{~b}$. The methyl group substituent in 11b reduces its polarity. It is also noted that $11 \mathrm{c}$ is more polar than 11d due to the methyl substituent in the aryl moiety of the sulfone group in 11d. These observations coincide very well with the data obtained (Tables 1 and 2). Similarly, in the second and third groups of the studied compounds (oximes and hydrazones, respectively), due to the methyl substituent, compound $16 \mathrm{a}$ is more polar than $16 \mathrm{~b}$ and compound $17 \mathrm{a}$ is more polar than compound $17 \mathrm{~b}$.

The linear regression parameters between $\log k$ values and methanol volume fraction are abridged in Table 2.

\section{Correlation between $\log K_{w}$ and $\varphi_{0}$ and computed $\log P$ values}

The following step in this work is to compare chromatographically-obtained lipophilicity parameters; $\log \mathrm{k}_{\mathrm{w}}$ or $\varphi_{0}$ of the studied compounds with clog $\mathrm{P}$ calculated using different computational methods (that utilize different theoretical approaches, principles of which were described by Mornar et al. [26]) such as ACD/Labs, miLOGP, ALOGPS, and APLOGP. A very good correlation exists between $\log \mathrm{k}_{\mathrm{w}}$ and computed $\log \mathrm{P}$ values ( $\mathrm{r}$ in all cases $\geq 0.8283$ ) as represented in Table 3, which confirms the reliability of HPLC technique as a tool for lipophilicity estimation. Although a fair correlation exists between $\varphi_{0}$ and miLogP, a good correlation still presents between $\varphi_{0}$ and the other computed $\log \mathrm{P}$ values (Table 3), which is a further proof for the validity of this approach for determination of lipophilicity. All the investigated compounds purity was confirmed by the $\mathrm{H}^{1} \mathrm{NMR}$ spectrometer (Table 4).

For further investigation of the differences among the computational and experimental lipophilicity, the 
Table 3 Correlation between experimental $\log \mathbf{k}_{\mathrm{w}}$ values or $\varphi_{0}$ values and computed log $\mathrm{P}$ values (calculated using different techniques)

\begin{tabular}{|c|c|c|c|c|c|c|}
\hline Compound & $\log k_{w}$ & $\varphi_{0}$ & miLog $\mathrm{P}^{\mathrm{a}}$ & ALOGPS $^{\mathbf{b}}$ & ACD/Labs ${ }^{c}$ & APLOGPC \\
\hline $11 a$ & 2.59 & 64.83 & 1.96 & 2.20 & 2.28 & 2.07 \\
\hline $11 b$ & 3.60 & 69.81 & 2.40 & 2.60 & 2.74 & 2.57 \\
\hline $11 \mathrm{c}$ & 3.15 & 75.14 & 2.76 & 3.05 & 3.04 & 2.92 \\
\hline $11 d$ & 4.78 & 77.99 & 3.21 & 3.38 & 3.5 & 3.33 \\
\hline $16 a$ & 1.94 & 64.39 & 2.02 & 1.86 & 2.42 & 2.62 \\
\hline $16 b$ & 2.72 & 72.57 & 2.47 & 1.86 & 2.88 & 2.86 \\
\hline $17 a$ & 4.59 & 77.97 & 5.33 & 4.37 & 4.12 & 3.83 \\
\hline $17 b$ & 5.18 & 74.52 & 5.78 & 4.60 & 4.58 & 4.09 \\
\hline Celecoxib & 4.18 & 85.51 & 3.61 & 3.99 & 4.61 & 3.92 \\
\hline \multicolumn{3}{|c|}{$\begin{array}{l}\text { r between log } k_{w} \text { values and computed log P } \\
\text { values }^{d}\end{array}$} & 0.8397 & 0.9107 & 0.8501 & 0.8283 \\
\hline \multicolumn{3}{|c|}{$r$ between $\varphi_{0}$ values and computed log $P$ values ${ }^{d}$} & 0.5548 & 0.7263 & 0.8414 & 0.8143 \\
\hline
\end{tabular}

Correlation between experimental $\log \mathrm{k}_{\mathrm{w}}$ values or $\varphi_{0}$ values and computed log $\mathrm{P}$ values (calculated using different techniques)

a Calculated using molinspiration online service (http://www.molinspiration.com/)

b Calculated using ALOGPS 2.1 online service (http://www.vcclab.org/lab/alogps/)

c Calculated using ACD/Labs online service (https://ilab.acdlabs.com/iLab2/)

${ }^{d}$ Correlation coefficient

Table 4 Purity of the studied compounds

\begin{tabular}{|c|c|}
\hline Compound number & Purity $^{a}$ \\
\hline $11 \mathrm{a}$ & 97.03 \\
\hline $11 \mathrm{~b}$ & 98.25 \\
\hline $11 \mathrm{c}$ & 94.27 \\
\hline $11 \mathrm{~d}$ & 96.84 \\
\hline $16 a$ & 99.50 \\
\hline $16 \mathrm{~b}$ & 98.10 \\
\hline $17 \mathrm{a}$ & 98.80 \\
\hline $17 \mathrm{~b}$ & 99.70 \\
\hline
\end{tabular}

${ }^{a}$ The purity of the target compounds was confirmed by $\mathrm{H}^{1} \mathrm{NMR}$ using Bruker NMR spectrometer and DMSO as solvent. Charts describing compounds $16 \mathrm{a}, \mathrm{b}$ and $17 \mathrm{a}, \mathrm{b}$ were included to confirm the purity of the mentioned compounds. HPLC run was also performed to check and evaluate such purity as well

lipophilicity values were arranged in a matrix of dimensions nine (compounds) $\times$ six $(2$ computational and four experimental lipophilicity $\left(\log \mathrm{k}_{\mathrm{w}}\right.$ or $\left.\varphi_{0}\right)$ ), then principal component analysis PCA was performed on the whole matrix.

The first principal component PC1 accounts for $86.7 \%$ of the data variation, whereas PC2 explains $8.1 \%$ of the data variation, respectively ( $94.8 \%$ total). As given in Fig. 3, the variation in the lipophilicity of the investigated analogues can be mainly explained by $\mathrm{PC} 1$, while $\mathrm{PC} 2$ explains differences among chromatographic and computational techniques, which strongly justify the utilization of chromatographic techniques for lipophilicity estimation as a promising substitute to experimental methods based on Shake-Flask method.

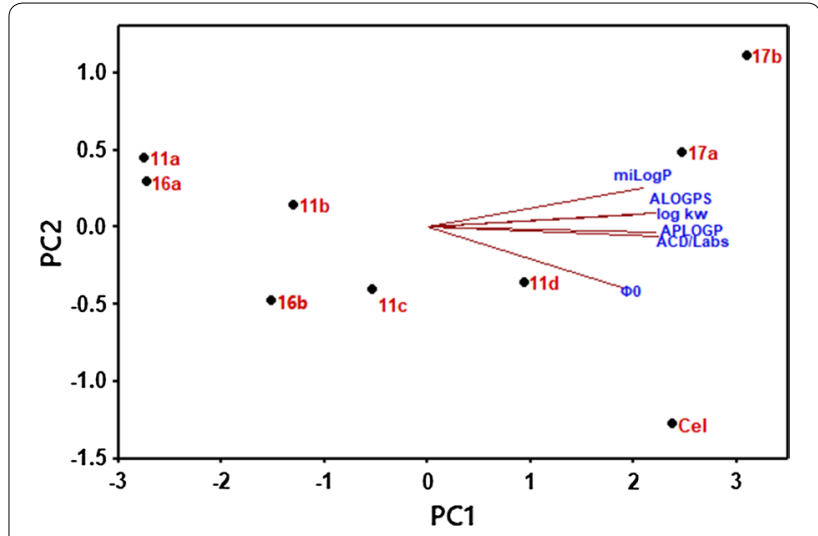

Fig. 3 Scores and loadings of Principal Component Analysis of the lipophilicity matrix

\section{Conclusion}

In this work, determination of lipophilicity of celecoxib and its newly synthesized analogues was carried out by RP-HPLC using a $\mathrm{C}_{18}$ silanized silica gel stationary phase. This experimental study proved to be simple, reliable and does not need sophisticated or complicated methodologies which show the importance of this method for pharmaceutical applications. Lipophilicity is an essential physicochemical property that affects pharmacokinetics and pharmacodynamics as well as toxicity of drug molecules. Moreover, it is well known that this essential property is important for the in vivo distribution of organic compounds by influencing their solubility, oral absorption, cell uptake, blood-brain 
penetration and metabolism and to rationalizing a number of biological events as membrane penetration and permeability. Under suitable isocratic chromatographic conditions, extrapolated retention factors are in good correlation with the computationally calculated $\log \mathrm{P}$ obtained using different software programs. Hence, RP-HPLC is an easily applicable technique for determination of lipophilicity and the chromatographic retention.

\section{Additional file}

Additional file 1. H-NMR for the synthesized compounds.

\section{Abbreviations}

RP-HPLC: reversed-phase high performance liquid chromatography; $\log P_{\text {ow: }}$ octanol-water partition coefficient; $\mathrm{K}$ : retention factor; $\varphi_{0}$ : the chromatographic index of hydrophobicity.

\section{Authors' contributions}

$\mathrm{FB}$ and $\mathrm{GH}$ planned the whole work. AR, HA and HE participated in the proposal for the method and literature review. FB, GH and JN supervised the experimental work and participated in the assay design. HE carried out the practical part, collects the results and wrote the paper. All authors read and approved the final manuscript.

\section{Funding}

No funding supporting this research.

\section{Availability of data and materials}

The data material are available whenever its needed from authors.

\section{Competing interests}

The authors declare that they have no competing interests.

\section{Author details}

${ }^{1}$ Department of Pharmaceutical Analytical Chemistry, Faculty of Pharmacy, Mansoura University, Mansoura 35516, Egypt. ${ }^{2}$ Department of Pharmaceutical Analytical Chemistry, Faculty of Pharmacy, Assiut University, Assiut 71526, Egypt. ${ }^{3}$ Department of Medicinal Chemistry, Faculty of Pharmacy, Mansoura University, Mansoura 35516, Egypt. ${ }^{4}$ Department of Applied Organic Chemistry, National Research Centre, Dokki 12622, Egypt.

Received: 26 March 2019 Accepted: 29 June 2019

Published online: 09 July 2019

\section{References}

1. Marciniec K, Bafeltowska J, Maślankiewicz MJ, Buszman E, Boryczka S (2016) Determination of the lipophilicity of quinolinesulfonamides by reversed-phase HPLC and theoretical calculations. J Liq Chromatogr Relat Technol 39(15):702-709

2. Duffus JH, Nordberg M, Templeton DM (2007) Glossary of terms used in toxicology, (IUPAC Recommendations 2007). Pure Appl Chem 79(7):1153-1344

3. Ayouni L, Cazorla G, Chaillou D, Herbreteau B, Rudaz S, Lantéri P et al (2005) Fast determination of lipophilicity by HPLC. Chromatographia 62(5):251-255

4. Giaginis C, Tsantili-Kakoulidou A (2007) Current state of the art in HPLC methodology for lipophilicity assessment of basic drugs. A review. J Liq Chromatogr Relat Technol 31(1):79-96

5. Santos Á, Soares JX, Cravo S, Tiritan ME, Reis S, Afonso C et al (2018) Lipophilicity assessement in drug discovery: experimental and theoretical methods applied to xanthone derivatives. J Chromatogr B. 1072(Supplement C):182-192
6. Andrić F, Héberger K (2015) Towards better understanding of lipophilicity: assessment of in silico and chromatographic logP measures for pharmaceutically important compounds by nonparametric rankings. J Pharm Biomed Anal 115(Supplement C):183-191

7. Lipinski CA, Lombardo F, Dominy BW, Feeney PJ (1997) Experimental and computational approaches to estimate solubility and permeability in drug discovery and development settings. Adv Drug Deliv Rev 23:3-25

8. OECD (1995) Test No. 107, Partition coefficient(n-octanol/water), Shake flask method. OECD, Paris

9. OECD (2005) Test No. 123, Partition coefficient(n-octanol/water), Slowstirring method. OECD, Paris

10. OECD (2004) Test No. 117, Partition coefficient(n-octanol/water), HPLC method. OECD, Paris

11. Hawry AM, Popiołek LP, Hawrył MA, Ryszard HS, Niejedli MA (2015) Chromatographic and calculation methods for analysis of the lipophilicity of newly synthesized thiosemicarbazides and their cyclic analogues 1,2,4-triazol-3-thiones. J Brazil Chem Soc 26(8):1617-1624

12. Rudraraju AV, Amoyaw PNA, Hubin TJ, Khan MOF (2014) Determination of $\log$ P values of new cyclen based antimalarial drug leads using RP-HPLC. Pharmazie 69:655-662

13. Marciniec K, Bafeltowska J, Maślankiewicz M, Buszman E, Boryczka S (2016) Determination of the lipophilicity of quinoline sulfonamides by reversed-phase HPLC and theoretical calculations. J Liqu Chromatogr Relat Technol 39(1):702-709

14. Tuzimski T, Sztanke K (2005) Retention data for some carbonyl derivatives of imidazo $[2,1-c][1,2,4]$ triazine in reversed-phase systems in TLC and HPLC and their use for determination of lipophilicity. Part 1. Lipophilicity of 8-aryl-3-phenyl-6, 7-dihydro-4H-imidazo [2,1-c][1,2,4] triazin-4-ones. JPC J Plan Chromatogr Mod TLC. 18(104):274-281

15. Moffat ACO, David M, Widdop B, Watts J (2011) Clarke's analysis of drugs and poisons, 4th edn. Pharmaceutical press, London

16. Sweetman SC (2014) Martindale: the complete drug reference, 38th edn. Pharmaceutical Press, London

17. Miles S (2007) Celecoxib.* xPharm: the comprehensive pharmacology reference. Elsevier, New York, pp 1-7

18. Bennett JS, Daugherty A, Herrington D, Greenland P, Roberts H, Taubert KA (2005) The use of nonsteroidal anti-inflammatory drugs (NSAIDs). A science advisory from the American Heart Association. Circulation $111(13): 1713-1716$

19. Abdel-Aziz HA, Al-Rashood KA, ElTahir KEH, Suddek GM (2014) Synthesis of $\mathrm{N}$-benzenesulfonamide-1H-pyrazoles bearing arylsulfonyl moiety: novel celecoxib analogs as potent anti-inflammatory agents. Eur J Med Chem. 80:416-422

20. Snyder LR, Kirkland JJ, Dolan JW (2011) Introduction to modern liquid chromatography. Wiley, Hoboken

21. Haber P, Baczek T, Kaliszan R, Snyder LR, Dolan JW, Wehr CT (2000) Computer simulation for the simultaneous optimization of any two variables and any chromatographic procedure. J Chromatogr Sci 38(9):386-392

22. Sima IA, Kot-Wasik A, Wasik A, Namieśnik J, Sârbu C (2017) Assessment of lipophilicity indices derived from retention behavior of antioxidant compounds in RP-HPLC. Molecules 22(4):550

23. Volná T, Motyka K, Hlaváč J (2016) Potential of high-performance liquid chromatography for distribution coefficient determination of 3-hydroxyquinolin-4(1H)-one derivatives. Chromatographia 79(17):1153-1163

24. Valkó K, Slégel P (1993) New chromatographic hydrophobicity index $\left(\varphi_{0}\right)$ based on the slope and the intercept of the log $k^{\prime}$ versus organic phase concentration plot. J Chromatogr A 631(1):49-61

25. Jevrić LR, Karadžić MŽ, Mandić Al, Podunavac Kuzmanović SO, Kovačević SZ, Nikolić AR et al (2017) Lipophilicity estimation and characterization of selected steroid derivatives of biomedical importance applying RP HPLC. J Pharm Biomed Anal 134:27-35

26. Mornar A, Damic A, Nigovic B (2011) Pharmacokinetic parameters of statin drugs characterized by reversed phase high-performance liquid chromatography. Anal Lett 44:1009-1020

\section{Publisher's Note}

Springer Nature remains neutral with regard to jurisdictional claims in published maps and institutional affiliations. 